OPEN ACCESS

Edited by:

Rubén N. Muzio,

Consejo Nacional de Investigaciones Científicas y Técnicas (CONICET),

Argentina

Reviewed by:

Rachele Mariani,

Sapienza University of Rome, Italy

Liang-Jen Wang,

Kaohsiung Chang Gung Memorial

Hospital, Taiwan

*Correspondence:

Shota Nishitan

nshota@u-fukui.ac.jp

Akemi Tomoda

atomoda@u-fukui.ac.jp

Specialty section: This article was submitted to

Health Psychology,

a section of the journal

Frontiers in Psychology

Received: 24 September 2021 Accepted: 30 November 2021

Published: 21 December 2021

Citation:

Kurata S, Hiraoka $D$

Ahmad Adlan AS, Jayanath $S$,

Hamzah N, Ahmad-Fauzi A,

Fujisawa TX, Nishitani S and

Tomoda A (2021) Influence of the

COVID-19 Pandemic on Parenting

Stress Across Asian Countries:

A Cross-National Study.

Front. Psychol. 12:782298.

doi: 10.3389/fpsyg.2021.782298

\section{Influence of the COVID-19 Pandemic on Parenting Stress Across Asian Countries: A Cross-National Study}

\author{
Sawa Kurata ${ }^{1,2,3}$, Daiki Hiraoka ${ }^{1,4}$, Aida Syarinaz Ahmad Adlan ${ }^{5}$, Subhashini Jayanath ${ }^{6}$, \\ Norhamizan Hamzah7 ${ }^{7}$, Aishah Ahmad-Fauzi', Takashi X. Fujisawa ${ }^{1,2,8}$, \\ Shota Nishitani ${ }^{1,2,8 *}$ and Akemi Tomoda ${ }^{1,2,3,8 *}$
}

${ }^{1}$ Research Center for Child Mental Development, University of Fukui, Fukui, Japan, ${ }^{2}$ Division of Developmental Higher Brain Functions, United Graduate School of Child Development, Osaka University, Kanazawa University, Hamamatsu University School of Medicine, Chiba University, and University of Fukui, Osaka, Japan, ${ }^{3}$ Department of Child and Adolescent Psychological Medicine, University of Fukui Hospital, Fukui, Japan, ${ }^{4}$ Japan Society for the Promotion of Science, Tokyo, Japan, ${ }^{5}$ Department of Psychological Medicine, Faculty of Medicine, University of Malaya, Kuala Lumpur, Malaysia, ${ }^{6}$ Department of Pediatrics, University of Malaya, Kuala Lumpur, Malaysia, ${ }^{7}$ Department of Rehabilitation Medicine, University of Malaya, Kuala Lumpur, Malaysia, ${ }^{8}$ Life Science Innovation Center, Faculty of Medical Sciences, University of Fukui, Fukui, Japan

Background: In a previous study, we demonstrated that the accumulation of parenting stress during prolonged school closures and restrictions on daily activities due to the COVID-19 pandemic in Japan indicates the need for mental health intervention for parents at higher risk of parenting stress. However, few studies have focused on parenting stress in other Asian countries, although they have experienced higher numbers of infections. The aim of the present study was to investigate whether parenting stress among caregivers increased across Asia due to school closures and restrictions on activities during the COVID-19 pandemic and to examine whether there were any country-specific, cross-country, or cross-regional risk factors for increased parenting stress.

Methods: We conducted an online survey immediately after the number of new cases in India significantly increased (September-November 2020). We measured parenting stress, anxiety, and fear associated with the COVID-19 crisis, as evaluated by the Parenting Stress Index, Short-Form (PSI-SF), and the Coronavirus Anxiety Scale (CAS), across three Asian countries - India $(n=142)$, Malaysia $(n=69)$, and Japan $(n=182)$ in addition to the United States $(n=203)$. We also investigated whether respondents had adverse childhood experiences (ACE) as a risk factor for parenting stress.

Results: For all countries, we found significant increases in participants' current parenting stress levels, compared to what they recalled regarding their lives before COVID-19-related restrictions and school closures were enacted. Textual analysis qualitatively identified common terms related to parenting stress across all countries. We also found a statistical model that indicated ACE in parents was a critical risk factor for higher parenting stress via increasing anxiety and fear related to the pandemic. 


\begin{abstract}
Conclusion: These results indicate the need to improve the mental health of caregivers who are at risk for higher levels of parenting stress during the COVID-19 pandemic in Asian countries as well as Western countries. These results indicate that there is a need to improve the mental health of caregivers who are at risk for higher levels of parenting stress during the COVID-19 pandemic globally.
\end{abstract}

Keywords: Coronavirus Anxiety Scale (CAS), Parenting Stress Index (PSI), school closure, parenting stress, COVID-19, Adverse Childhood Experiences (ACE)

\section{INTRODUCTION}

On March 11, 2020, the outbreak of the novel coronavirus disease 2019 (COVID-19) which began in Wuhan, China in December 2019 was classified by the World Health Organization (WHO) (World Health Organization, 2021) as a pandemic, a situation that has persisted for more than a year. Since the beginning of the pandemic, many schools worldwide were closed for an average of 3.5 months in 2020 (UNICEF, 2021). As a result of these prolonged school closures, children have often shown tendencies toward mood swings, anxiety, emotional problems, and behavioral and cognitive changes (Fegert et al., 2020; Francisco et al., 2020; Jiao et al., 2020; Tso et al., 2020; Tang et al., 2021). Further, the restrictions on daily life due to school closures and those placed on activities affect not only children's mental health but also that of their caregivers (Cusinato et al., 2020).

Previously, we were the first to survey the impact of prolonged school closure due to the COVID-19 pandemic on the mental health of caregivers in Japan. Our survey of caregivers with children out of school revealed an increase in parenting stress compared to pre-pandemic levels. Further, qualitative analysis using textual mining of free descriptions regarding parenting stress during the pandemic indicated that spending a lot of time with their children was the primary stressor (Hiraoka and Tomoda, 2020). A high number of cumulative stressors in caregivers due to school closures and quarantine measures related to the pandemic has been shown to be associated with higher parenting stress, which indirectly becomes a potential risk factor for child maltreatment (Ramaswamy and Seshadri, 2020; Afrin and Zainuddin, 2021; Lee et al., 2021). Thus, several reports have indicated the importance of adequate mental health care for caregivers (Brown et al., 2020; Marchetti et al., 2020; Spinelli et al., 2020). As the pandemic shows no indications of ending soon, increased risk of parenting stress among caregivers, as described above, is an urgent issue that must be addressed in consideration of children's healthy development and lifelong physical and mental health of parents and children.

From August to September 2020, shortly after we reported the findings of our previous survey conducted in Japan, a large-scale outbreak occurred in India, unlike any sudden rise in cases during a pandemic seen before in Asia. As a result, India became the country with the second highest number of COVID-19 infections worldwide after the United States; then, the tension about the spread of COVID-19 increased. Subsequently, the number of cases in Malaysia began to increase around September 2020, before dramatically increasing starting around December 2020 and continuing to grow
(Supplementary Figure 1). Nevertheless, few epidemiological studies examining the influence of restrictions placed on activities during the pandemic, on the mental health of children and their caregivers have been conducted in Asian countries, except for China, as there had not yet been large-scale outbreaks such as those in Europe and the United States. However, the Indian government declared a state of emergency in March 2020, and imposed school closures and restrictions on activities for 7 months, which was twice as long as the global average. In Malaysia, a movement control order (MCO) was declared in March 2020. School closures and restrictions on activities have been repeatedly imposed since, to varying extents. Recently, a state of emergency was declared and continues to be in operation in Malaysia. Although the situation has been very serious, with many severe cases and deaths, the social situation of children and caregivers in these Asian countries seems to have been similar to that in Japan, Europe, and the United States.

In addition to biological vulnerabilities, such as psychiatric disorders, brain substrates, and genetic and epigenetic variations among caregivers themselves, psychological and social vulnerabilities such as poverty, single parenting, lack of social support, and the caregivers' own childhood experiences of maltreatment can also be significant risk factors for engaging in child maltreatment in the context of situations where parenting stress is exceptionally high (Bowers and Yehuda, 2016; Shimada et al., 2018, 2019; Hiraoka and Nomura, 2019; Park et al., 2019; Cecil et al., 2020; Kuboshita et al., 2020; Hiraoka et al., 2021; Kasaba et al., 2021). One vital psychological and social risk factor for engaging in child maltreatment, regardless of country, ethnicity, or culture, is when caregivers themselves had adverse childhood experiences (ACE) (Felitti et al., 1998). It has been reported that the more adversity experienced in childhood, the higher the vulnerability to stress in adulthood (Albott et al., 2018). Even in the absence of unprecedented circumstances such as a pandemic, child-rearing often involves stress; thus, caregivers who experience more adversity in childhood may be at higher risk of developing child-rearing difficulties (Lange et al., 2019). Individuals who have experienced childhood adversity have been shown to exhibit mental vulnerability to the large-scale social change of COVID-19 (Guo et al., 2020). In addition to the ACE, a higher level of anxiety related to COVID-19 in caregivers may also be an additional contributing risk factor to increased parenting stress. However, since this high anxiety level may interact with caregivers' ACE, it is also necessary to consider this structure when comprehensively investigating increases in parenting stress. 
Therefore, the present study first aimed to investigate whether parenting stress among caregivers increased across Asia, as it did in Europe and the United States (Brown et al., 2020; Calvano et al., 2021; Lee et al., 2021), due to school closures and restrictions on activities during the COVID-19 pandemic. We chose India, which experienced an outbreak in August and September 2020 and had the world's second highest number of infections, to represent South Asia; Malaysia, where the first rise in cases occurred after September 2020 and is still increasing, to represent Southeast Asia; and Japan, which was the target country of our longitudinal survey based on our previous study (Hiraoka and Tomoda, 2020), to represent East Asia. The United States, which has the world's largest number of infections and is ethnically and culturally different from regions in Asia, was chosen as the reference country for comparison. The second aim of the present study was to examine whether there were any country-specific, cross-country, or cross-regional risk factors for increased parenting stress. Finally, the third aim was to clarify how childhood adversity is related to COVID-19 anxiety as a common risk factor for high parenting stress.

\section{MATERIALS AND METHODS}

\section{Data Collection}

We collected data across the three Asian countries examined in this study-India, Malaysia, and Japan as representative cases for South, Southeast, and East Asia, respectively-in addition to the United States as a reference country. Participants from CrowdWorks (Japan) and Amazon's Mechanical Turk (MTurk) (India and the United States) online worker pools provided consent for participation prior to beginning the survey on the impact of COVID-19 on parenting stress. We used pre-screening filters offered by the platforms to limit participant age (1855 years; MTurk only), parenthood status, and location (Japan, India, and the United States). All information gathered was processed anonymously, and participants received an incentive of US\$1.50. The survey was conducted entirely on the web via psyToolkit (Stoet, 2010, 2017) on any platform between September 28th to October 21st, 2020, approximately 1 week after the number of new cases rapidly increased in India. Responses from the same ID and those with the exact same free text, which were thought to be from the same individual, were excluded (39 in India, 4 in Japan, and 8 in the United States), as shown in Table 1. We simultaneously conducted an extra survey in Malaysia from September to November 2020, when the number of new cases had begun to gradually increase for the first time, as it would be valuable to prospectively capture the live dynamics of parenting stress from the beginning. Unlike the other countries where we used online worker pools, in Malaysia, participants were recruited as volunteers. All participants provided informed consent before the survey. The survey was conducted in a similar manner to those in India and the United States via psyToolkit (Stoet, 2010, 2017). The study protocol and all procedures were approved by the Ethics Committee of the University of Fukui, Japan (Assurance \#FU-20200007).

\section{Psychological Questionnaires}

The Parenting Stress Index ${ }^{\mathrm{TM}}$, Third Edition, Short Form (Abidin, 1995) (PSI-SF) was used to measure parenting stress. Each item of the PSI-SF is rated on a five-point scale, ranging from 1 (strongly disagree) to 5 (strongly agree). We used three subscales which assess different types of parenting stress: Parental Distress (PD), the extent to which parents feel competent, restricted, conflicted, supported, and/or depressed in their role as a parent; Parent-Child Dysfunctional Interaction (P-CDI), the extent to which parents feel satisfied with their child and their interactions with them; and Difficult Child (DC), how a parent perceives their child to be, whether the child is easy or difficult to take care of, in addition to the total stress which is an indication of overall level of stress a person is feeling in their role as a parent. Participants were asked to complete the PSI-SF twice. First, the participants answered the PSI-SF without any particular instructions. Then, following completion, they were asked to complete the PSI-SF again, recalling what it was like before school closures and restrictions on activities. Such retrospective measuring methods have often been used when examining the health effects of COVID-19 (Gao and Scullin, 2020; Robillard et al., 2021).

In addition to the PSI-SF, we used the Coronavirus Anxiety Scale (CAS) to evaluate excessive concern and dysfunctional anxiety symptoms associated with the COVID-19 pandemic (Lee, 2020). Each item of the CAS is rated on a five-point scale, ranging from 0 (not at all) to 4 (nearly every day), based on experiences over the past 2 weeks. A CAS total score $\geq 9$ indicates probable dysfunctional coronavirus-related anxiety (Lee, 2020). Elevated scores on a particular item may indicate problematic symptoms that could warrant further assessment and/or treatment.

Whether participants had ACE was determined based on their responses to the questions from the Centers for Disease Control (CDC)'s 2011 Behavioral Risk Factor Surveillance System (BRFSS) questionnaire, which contains 11 questions to measure three types of child abuse (physical, sexual, and emotional) and five types of household dysfunction (substance abuse, mental illness, domestic violence, incarceration/jail, and divorce/separation) (Merrick et al., 2018). Self-reported exposure to any single ACE category is counted as one point toward the final ACE score (range: $0-8$ ).

\section{Qualitative Measurement of Parenting Stress}

Participants were also asked to freely describe any parenting stress they were currently experiencing ("Are you experiencing parenting stress due to the spread of COVID-19? What kind of stress are you feeling as a result of school closures?"). Although this question was not compulsory, $89 \%$ of participants answered it. While participants from India, Malaysia, and the United States answered this question in English, Japanese participants answered it in Japanese. We analyzed the text data, which was translated into English via Google Translate for Japanese cases. 
TABLE 1 | Demographics of the participants.

\begin{tabular}{|c|c|c|c|c|}
\hline & India, $N=142^{\mathrm{a}}$ & Malaysia, $N=67^{a}$ & Japan, $N=182^{a}$ & United States, $N=203^{a}$ \\
\hline Age (year) & $32.5(6.8)$ & $39.4(4.7)$ & $37.4(6.4)$ & $36.5(8.7)$ \\
\hline Female & $51(35.9)$ & $48(71.6)$ & $145(79.7)$ & $120(59.1)$ \\
\hline Number of children & $2.2(3.4)$ & $2.4(1.1)$ & $1.6(0.6)$ & $1.7(1.3)$ \\
\hline \multicolumn{5}{|l|}{ Cohabitation } \\
\hline Children (single) & $32(22.5)$ & $6(10.7)$ & $6(3.4)$ & $40(19.7)$ \\
\hline Children + spouse/partner & $46(32.4)$ & $35(62.5)$ & $157(88.2)$ & $116(57.1)$ \\
\hline Children + spouse/partner + parent/others & $64(45.1)$ & $15(26.8)$ & $15(8.4)$ & $47(23.2)$ \\
\hline \multicolumn{5}{|l|}{ Race } \\
\hline Asian (including Indian) & 139 (97.9) & $53(98.1)$ & $182(100.0)$ & $7(3.4)$ \\
\hline Caucasian & $0(0.0)$ & $0(0.0)$ & $0(0.0)$ & $166(81.8)$ \\
\hline Other & $3(2.1)$ & $1(1.9)$ & $0(0.0)$ & $30(14.8)$ \\
\hline Ethnicity (Hispanic or latino/a) & NA & NA & NA & $23(11.3)$ \\
\hline Education (> graduated college) & $138(97.2)$ & $50(74.6)$ & NA & $175(86.2)$ \\
\hline \multicolumn{5}{|l|}{ House hold income (per year) } \\
\hline$<\$ 30,000$ & $89(62.7)$ & $40(59.7)$ & $25(13.7)$ & $34(16.7)$ \\
\hline$\$ 30,000-\$ 75,000$ & $38(26.8)$ & $10(14.9)$ & $129(70.9)$ & $96(47.3)$ \\
\hline$\$ 75,000<$ & $15(10.6)$ & $17(25.4)$ & $28(15.4)$ & $73(36.0)$ \\
\hline
\end{tabular}

a Statistics presented: mean (SD); n (\%). NA, not applicable.

\section{Statistical Analysis}

To investigate the effects on parenting stress from prolonged school closures and restraints on activities due to the pandemic in each country, a two-way mixed ANOVA was conducted. Considering the literatures on the COVID-19 pandemic highlights a greater impact on the female population (Lebel et al., 2020; Grumi et al., 2021; Malkawi et al., 2021), we also conducted a three-way mixed ANOVA to explore gender differences were existing. Additionally, to visualize the characteristics of the frequently reported words and the similarities or differences among countries, we conducted co-occurrence network analysis using KH-Coder (Higuchi, 2016, 2017). Words were extracted from free descriptions of parenting stress, and the top 60 words that occurred most frequently were extracted. In the cooccurrence network analysis, words that were unique to each country were linked to the circle of the country. If a word was similarly extracted across countries (e.g., stress or time), that word was linked to the respective country circle. Pearson correlation analyses were conducted between each outcome to examine any associations between PSI, CAS, and ACE. We conducted mediation analysis to assess whether the CAS mediated the link between ACE and PSI. The indirect effect was tested by bootstrapping confidence intervals using the lavaan package (Rosseel, 2012) of the R statistical software program (R Core Team, 2019). The model parameters were set to give biascorrected $95 \%$ confidence intervals and to run 2,000 bootstrap resamples. Then, multi-group analysis was used to examine differences among countries in the path coefficients between PSI, CAS, and ACE. We compared the first (which allowed for the structural paths to vary across countries) and second models (which constrained the regression paths to remain the same for countries) to identify any country-related differences. In addition, to confirm if there were gender differences in the path coefficients among countries in the path analysis, we conducted multi-group analyses between men and women in each country. All statistical analyses were performed using R 3.6.1.

\section{RESULTS}

As shown in Table 2, a two-way mixed ANOVA for PSI-SF total scores revealed significant main effects for "country" $[F(3$, $\left.526)=32.7, p=2.16 \mathrm{E}-19, \eta^{2}=0.15\right]$ and "time" $[F(1,526)=20.8$, $\left.p=6.43 \mathrm{E}-06, \eta^{2}=0.002\right]$ in all the sub-scales and the total score. However, no significant interactions between "country" and "time" were observed for all cases $[F(3,526)=0.9, p=0.45$, $\left.\eta^{2}=0.0002\right]$. Pairwise $t$-tests of PSI-SF total scores between each "country," irrespective of the "time," revealed India was the highest compared to the other countries (vs. Malaysia: $p=6.9 \mathrm{E}-$ 22 , vs. Japan: $p=1.2 \mathrm{E}-24$, and vs. United States: $p=0.02 ; p$-values were adjusted using the Bonferroni multiple correction method). All other combinations of the pairwise $t$-tests between each "country" were also significant (Malaysia vs. Japan: $p=9.55 \mathrm{E}-3$, Malaysia vs. United States: $p=0.02$, and Japan vs. United States: $p=2.59 \mathrm{E}-16)$. No significant main effect for gender difference and interactions between gender and the other factors across the countries was found $\left[F(1,517)=1.59, p=0.21, \eta^{2}=0.003\right]$. Five participants were excluded from the analysis due to no gender assignment information available.

The results of the co-occurrence network analysis for openended statements regarding parenting stress are shown in Supplementary Figure 2. Commonly, "child" and "school," which may be related to school closure, were reported as parenting stressors in each country. In addition, the word "time" was also found for all countries. While there were some positive comments, such as that school closures allowed children to spend more time at home and for parents to spend more time with their children, there were also several comments indicating that 
TABLE 2 | The results of the psychological questionnaires.

\begin{tabular}{|c|c|c|c|c|c|c|}
\hline & Time & India, $N=139$ & Malaysia, $N=39$ & Japan, $N=155$ & United States, $N=197$ & Statistics* \\
\hline \multicolumn{7}{|c|}{ PSI-SF } \\
\hline \multirow[t]{2}{*}{ PD } & Before & $38.0(13.6)$ & $23.2(7.7)$ & $29.3(9.2)$ & $35.1(12.6)$ & \multirow{2}{*}{$F(1,526)=18.7, P=1.81 \mathrm{E}-05, \eta^{2}=0.003$} \\
\hline & After & $39.3(12.6)$ & $25.2(7.5)$ & $30.5(8.7)$ & $36.6(12.0)$ & \\
\hline \multirow[t]{2}{*}{ P-CDI } & Before & $35.2(14.2)$ & $20.6(6.9)$ & $22.7(6.4)$ & $32.0(13.4)$ & \multirow{2}{*}{$F(1,526)=5.2, P=0.023, \eta^{2}=0.0005$} \\
\hline & After & $35.2(13.0)$ & $22.1(7.0)$ & $23.8(6.4)$ & $32.0(13.0)$ & \\
\hline \multirow[t]{2}{*}{ DC } & Before & $35.2(12.8)$ & $22.5(7.8)$ & $27.3(9.6)$ & $33.7(12.4)$ & \multirow{2}{*}{$F(1,526)=16.9, P=4.63 \mathrm{E}-05, \eta^{2}=0.002$} \\
\hline & After & $36.0(12.1)$ & $23.7(7.1)$ & $29.2(9.0)$ & $34.6(12.1)$ & \\
\hline \multirow[t]{2}{*}{ Total } & Before & 108.5 (39.3) & $66.3(20.3)$ & $79.3(22.2)$ & $100.8(36.7)$ & \multirow{2}{*}{$F(1,526)=20.8, P=6.43 \mathrm{E}-06, \eta^{2}=0.002$} \\
\hline & After & $110.5(35.7)$ & 71.0 (19.5) & 83.4 (21.3) & $103.2(34.8)$ & \\
\hline CAS & - & $7.5(5.6)$ & $0.6(1.4)$ & $2.3(3.7)$ & $5.3(5.3)$ & \\
\hline ACE & - & $3.3(2.1)$ & $0.8(1.1)$ & $1.6(1.6)$ & $3.1(2.2)$ & \\
\hline
\end{tabular}

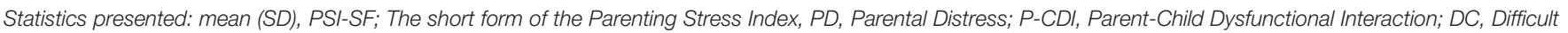
Child; CAS, COVID-19 anxiety scale; ACE, Adverse childhood experience. *Two-way mixed ANOVA main effect of "Time."

parents did not have time to relieve their own stress (e.g., "I am stressed a lot more because my kids are ALWAYS here. Them being here all the time bring more chores, bills, and less me time. I never get privacy anymore just like when they were toddlers."). This suggests that it may be necessary to find ways for parents to secure time for themselves in the limited space of the home to maintain their mental health during the pandemic.

Pearson correlation analyses between the PSI-SF (including sub-scales), CAS, and ACE across all countries revealed they were robustly correlated with each other (Supplementary Figure 3). For example, total PSI-SF scores were significantly correlated with CAS scores $(r=0.61, p=5.5 \mathrm{E}-59)$, and ACE $(r=0.53, p=3.7 \mathrm{E}-42)$. All within-country combinations were also significant.

We designed the mediation model with CAS as a mediating variable, ACE as an explanatory variable, and PSI as an outcome variable. In this model, the paths from ACE to CAS (a) and from CAS to PSI (b) were significant $(a=1.05, \mathrm{SE}=0.09, p=2 \mathrm{E}$ $16 ; b=2.97, \mathrm{SE}=0.27, p=2 \mathrm{E}-16)$, and the total effect was also significant (total effect $=8.13, \mathrm{SE}=0.56, p=2 \mathrm{E}-16$ ). There was a significant indirect effect [indirect effect $=3.11, \mathrm{SE}=0.38$, $95 \% \mathrm{CI}=(2.41,3.88)]$. Furthermore, the direct effect of ACE on PSI was until significant after addition of the mediator (direct effect $=5.02, \mathrm{SE}=0.58, p=2 \mathrm{E}-16$ ), which indicated CAS had a partial indirect effect on PSI.

Finally, we ran a multi-group analysis to examine whether the path coefficients differed significantly between countries (Figure 1). A model with no constraints on the path coefficients and a model with equality constraints were compared. The significant chi-square differences indicated that the regression coefficient differed by country $\left[\chi^{2}(9)=47.7, p=2.9 \mathrm{E}-07\right]$. The AIC of the former model (8530.8) was smaller than that of the latter model (8560.5), and the model without constraints was adopted. In both India and the United States, the coefficients between each variable were high, indicating that ACE was closely related to anxiety about COVID-19 and parenting stress. A similar relationship was found in Japan, but the path coefficients were not necessarily higher than India and United States, and ACE was not significantly associated with parenting stress. Finally, regarding Malaysia, the associations among each variable were not significant and the coefficients were smaller than the other countries. These results suggested that ACE may not necessarily be associated with anxiety and parenting stress in Malaysia. Or, there is another possibility that ACE may have been under-reported by participants due to the prevailing cultural norms in Malaysia. The multi-group analyses, in which a model with no constraints on the path coefficients and a model with equality constraints were compared between men and women in each country, showed no significant Chisquare differences indicating that the regression coefficient did not differ between gender [India: $\chi^{2}(3)=6.61, p=0.09$, Malaysia: $\chi^{2}(3)=3.52, p=0.32$, Japan: $\chi^{2}(3)=3.34, p=0.34$, and United States: $\chi^{2}(3)=6.41, p=0.09$ ].

\section{DISCUSSION}

This study examined the social and psychological risk factors contributing to increased parenting stress amidst the COVID19 pandemic, across countries. The results showed that, as we expected, parenting stress increased under the COVID-19 pandemic in all the Asian countries and the United States compared to the pre-pandemic period. This study is the first to concurrently examine and compare parenting stress in three different Asian countries. We also found that there was originally a difference in PSI scores among countries regardless of the period, which has been discussed in detail later. However, India had the highest PSI scores, followed by the United States of the sample countries. The mediation analysis showed that the number of ACE was strongly associated with higher parenting stress, and that anxiety and fear about the pandemic itself mediated the effect.

When we conducted this online survey in September-October 2020, the United States and India were the first and second most infected countries globally, while Malaysia and Japan had relatively fewer cases than other countries where COVID-19 was prevalent. Nevertheless, both Malaysia and Japan showed an increase in parenting stress during the pandemic. Other 

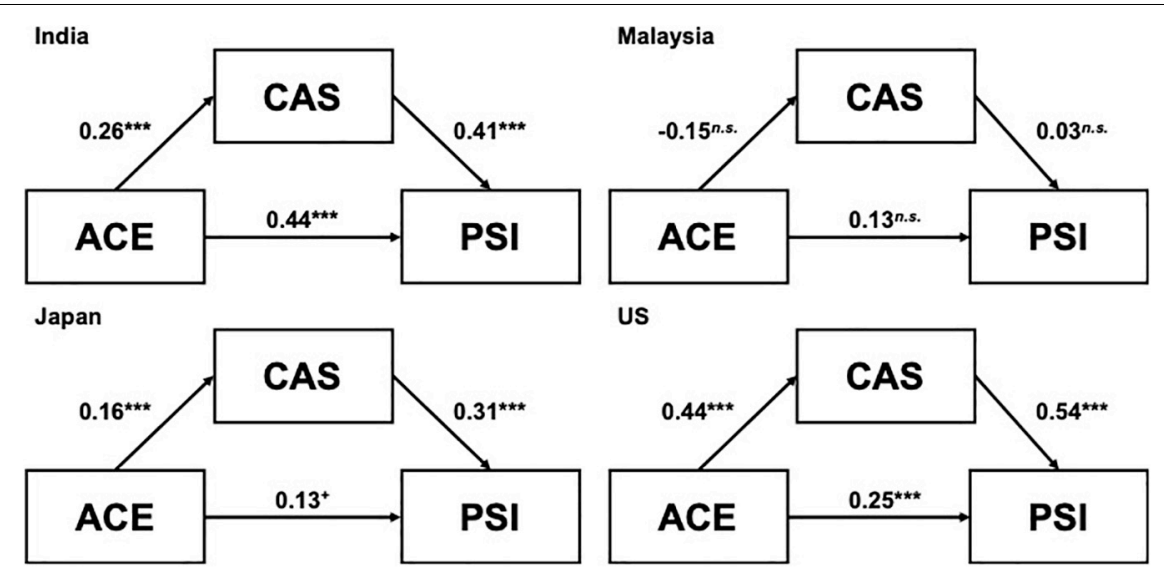

FIGURE 1 | The cross-cultural mediating relationships between adverse childhood experiences, anxiety about COVID-19, and parenting stress. The paths represent unstandardized regression coefficients. ACE, Adverse childhood experience; CAS, Coronavirus Anxiety Scale; PSI, Parenting Stress Index (Total score).

${ }^{* * *} p<0.001,{ }^{*} p<0.05,{ }^{+} p<0.10$.

studies conducted in Italy and Germany showed that parents experienced more parenting-related exhaustion due to social distancing as well as the closure of schools and child-care facilities, which manifested itself in increased parenting stress (Marchetti et al., 2020; Calvano et al., 2021). However, these studies were conducted in countries with high numbers of infections and deaths, unlike Malaysia and Japan. Therefore, the present study suggests that the cause of increased parenting stress is likely not only from factors directly related to the severity of the COVID-19 pandemic, such as the high number of infections and deaths but also other factors. Many Asian countries, including Malaysia and Japan, repeatedly declared a lockdown, MCO or state of emergency to combat the pandemic; schools were closed, activities were restricted, and many experienced social isolation (Nazif-Muñoz et al., 2021; Tang et al., 2021). It is to be noted that the social environment changed drastically due to unemployment and telecommuting (Lawson et al., 2020). The results of our textual analysis showed that the words "children," "school," and "time" were commonly reported as the specific stressors of the participants in every country. Although some participants viewed the situation in a positive light, reporting that they had "more family time since schools were closed and children spent more time at home," many viewed it negatively, saying that "they had less free time and did not have time to relieve their stress." The inflation of these inconveniences compared to the pre-pandemic era, and the maladaptation to new environmental changes, may have led to increased parenting stress. For example, Dickerson and Kemeny (2004) conducted a meta-analysis of the relationship between the characteristics of stressors and cortisol responses, and found that tasks containing uncontrollable elements were associated with robust cortisol reactivity (Dickerson and Kemeny, 2004). It is possible that in a situation where action is restricted worldwide, the physical symptoms of stress response, such as exhaustion, or changes in sleeping habits (Elhadi et al., 2021) are also seen, which may have led to an increase in parenting stress. However, there are some individual differences in tolerance to stressors. Individuals vulnerable to ambiguity are more stressed and rate their subjective well-being lower (Hancock and Mattick, 2020). The spread of COVID-19 can also be considered as an ambiguous situation, unlikely to be resolved immediately (Durodié, 2020), and the adaptation to this situation may be affected by individual differences. Further identification of factors may be necessary for the future.

We also found that childhood adversity as well as anxiety and fear about COVID-19 were risk factors for higher parenting stress during the pandemic. If high parenting stress is a risk factor for child maltreatment, our results support the well-known theory concerning the intergenerational cycle in survivors who were exposed to maltreatment during childhood that leads them to maltreat their child after becoming a parent (Lange et al., 2019; Uddin et al., 2020). In the mediation analysis, it was found that those with more ACE were more likely to have higher anxiety and fear about the COVID-19 pandemic. A model for predicting parenting stress has been suggested from childhood adversity, anxiety and fear about COVID-19. Numerous studies suggest that individuals who have experienced many childhood adversities, are vulnerable to hypothalamic pituitary adrenal (HPA) axis responses and a variety of other psychiatric disorders (Heim et al., 2000). Kalia et al. (2020) reported that individuals who were maltreated as children, but were not exposed to other social adversities such as poverty, were associated with fear of COVID-19, which resulted in higher anxiety. Moreover, given that a higher number of ACE is linked to greater susceptibility to parenting stress, this influence may occur with additional reinforcement in this unusual situation. The simultaneous multipopulation analysis revealed that this mediation model was not completely common in every country. Cultural differences may exist across countries. Guo et al. (2020) studied maternal mental health in China, Italy, and Netherlands under the impact of the current pandemic and reported the protective effect of grandparenting support and higher number of children, on mental health symptoms of Chinese mothers, but not Italian and Dutch mothers. Protective/risk factors on maternal mental health may differ according to each country's socio-cultural background. 
Thus, the relationship between ACE, anxiety and fear due to COVID-19, and parenting stress might be influenced by each country's socio-cultural background, including history, culture, ideology, and values. India and the United States, where the influence of ACE was profound, were the regions with the most significant spread of infections globally during the study (October 2020), where parents were more likely to be anxious about COVID-19. Those who had ACE and were highly vulnerable to stress were more likely to be affected emotionally by their childhood adversity in situations where the infection rate had substantially increased such as India and the United States. It is possible that the link between the ACE and anxiety and fear in these countries appeared stronger than in Malaysia and Japan. It appears that the pandemic situation and lockdown will continue intermittently. Above all, when the infection rates rise, social support and mental health care would be required, especially for individuals with more ACE than usual.

Furthermore, our data indicated that the original level of parenting stress differed in each country, and India had the highest level. In some areas in India, the standard of living remains low (International Monetary Fund, 2021); thus, economic poverty may have been directly related to high parenting stress. Furthermore, insufficient mental health care systems (Sharma et al., 2007), labor shortages, high population densities that make it easier for infections to spread, and shortages and price surges of face masks and other protective equipment may also have contributed to the high levels of parenting stress (Haque et al., 2020). Moreover, it was noticed that many of the respondents in India were men (64\%). Under the pandemic, men's burden of housework and childcare may have increased. In contrast, several studies have reported that COVID-19 has increased the psychological burden on Indian women (Gopal et al., 2020; Malhi et al., 2021). Traditionally, there is a longstanding concept of "patriarchy" in certain parts of India, a family structure in which the father has absolute power and control over family members. Therefore, Indian women and children tend to internalize distress from an early age, based on the socially accepted notion that externalizing feelings is unacceptable (Levey et al., 2017). The extra stress caused by the unexpected pandemic added to the original oppressive stress may result in a higher level of parenting stress than in other countries. Parenting stress in the United States was the second-highest after India, probably because the United States has been the world's leading country in terms of the number of infections and deaths for an extended period. This may lead to a high level of anxiety about the threat to life directly related to COVID-19 infection.

The present study has six major research limitations. First, it did not have a consistent online survey platform across countries. India and the United States used Amazon MTurk, Japan used CrowdWorks, and Malaysia did not use any online worker pool. As a result, there was a bias in the number of participants and their characteristics among the countries. Therefore, cautions should be taken when comparing our results from Malaysia with those of other countries. However, the online survey itself was effective as it allowed us to promptly collect a large amount of data in a short period because of the everchanging situation due to the COVID-19 pandemic and the period of social distancing and self-isolation. Second, the sample size of the present study was relatively small than the other previous studies. We started the present study based on our previous study in Japan (Hiraoka and Tomoda, 2020), and thus we have tried to match its sample size for the other countries. Third, we did not match age and gender across the countries. There is a report that MTurk workers are predominantly male (Djellel et al., 2018). In this instance, the percentage of males was high in India and the United States. Fourth, the evaluation instrument was a self-administered questionnaire, leading to a bias toward socially desirable answers. Although there is a problem of accuracy, since online behavioral experiments are now available (Stoet, 2010, 2017), a more objective evaluation could have been made if such experiments were utilized. Fifth, the assessment of parenting stress before the pandemic was conducted using a retrospective response method. Although the reliability of retrospective response methods may be questioned as the data is limited to individual data, it is more consistent at the population level (Lena et al., 2020). Therefore, we did not use retrospective response methods in our analysis except when comparing before and after the pandemic. Longitudinal studies in particular, should be conducted prospectively. However, as we had to capture response promptly due to the unpredictable situation, this method was unavoidable. Finally, the timing of the survey may influence the outcomes. Parenting stress may fluctuate depending on the situation of the infections at the time.

\section{CONCLUSION}

In the present study, we found that parenting stress increased in the three Asian countries and the United States during the COVID-19 pandemic. Higher number of ACE were strongly associated with higher parenting stress, an influential risk factor across countries. Anxiety and fear about the COVID19 pandemic also mediated the effect. ACE may lead to vulnerable parenting and trigger stress responses which induce child maltreatment, which can be aggravated by a negative unprecedented situation. In addition to examining the caregivers' current state, a retrospective assessment of past adversity experiences is warranted, because it can be expected to capture the risk of maltreatment more closely representative of the actual situation. A focus on ACE to provide more accurate support for parents and their evaluation should also be considered.

\section{DATA AVAILABILITY STATEMENT}

The raw data supporting the conclusions of this article will be made available by the authors, without undue reservation.

\section{ETHICS STATEMENT}

The studies involving human participants were reviewed and approved by the Ethics Committee of the University of Fukui, Japan (Assurance \#FU-20200007). The patients/participants provided their written informed consent to participate in this study. 


\section{AUTHOR CONTRIBUTIONS}

SK, DH, and SN designed the study. SK conducted interviews with supervision from $\mathrm{DH}$ and $\mathrm{SN}$. AA, SJ, NH, and AA-F collected the sample data from Malaysia. DH and SN completed all analysis and prepared tables and figures. SK wrote the main manuscript. Write up was completed by SK with critical revisions and supervision from DH and SN. All authors made substantial contributions to the analysis and data interpretation prior to agreeing upon key findings, conclusions, and approved the final manuscript.

\section{FUNDING}

All phases of this study were supported by AMED under Grant Number JP20gk0110052 (AT and SN), Grant-in-Aid for JSPS Fellows (20J002707 to DH), Grant-in-Aid for "Creating a Safe and Secure Living Environment in the Changing Public and Private Spheres" from the Japan Science and Technology

\section{REFERENCES}

Abidin, R. R. (1995). Parenting Stress Index, 3rd Edn. Odessa, FL: Psychological Assessment Resources.

Afrin, T., and Zainuddin, M. (2021). Spike in child marriage in Bangladesh during COVID-19: determinants and interventions. Child Abuse Negl. 112:104918. doi: 10.1016/j.chiabu.2020.10 4918

Albott, C. S., Forbes, M. K., and Anker, J. J. (2018). Association of childhood adversity with differential susceptibility of transdiagnostic psychopathology to environmental stress in adulthood. JAMA Netw. Open 1:e185354. doi: 10.1001/ jamanetworkopen.2018.5354

Bowers, M. E., and Yehuda, R. (2016). Intergenerational transmission of stress in humans. Neuropsychopharmacology 41, 232-244. doi: 10.1038/npp.20 15.247

Brown, S. M., Doom, J. R., Lechuga-Peña, S., Watamura, S. E., and Koppels, T. (2020). Stress and parenting during the global COVID19 pandemic. Child Abuse Negl. 110:104699. doi: 10.1016/j.chiabu.2020.10 4699

Calvano, C., Engelke, L., Di Bella, J., Kindermann, J., Renneberg, B., and Winter, S. M. (2021). Families in the COVID-19 pandemic: parental stress, parent mental health and the occurrence of adverse childhood experiences-results of a representative survey in Germany. Eur. Child Adolesc. Psychiatry Online ahead of print doi: 10.1007/s00787-021-01 739-0

Cecil, C. A. M., Zhang, Y., and Nolte, T. (2020). Childhood maltreatment and DNA methylation: a systematic review. Neurosci. Biobehav. Rev. 112, 392-409.

Cusinato, M., Iannattone, S., Spoto, A., Poli, M., Moretti, C., Gatta, M., et al. (2020). Stress, resilience, and well-being in italian children and their parents during the COVID-19 pandemic. Int. J. Environ. Res. Public Health 17:8297. doi: 10.3390/ijerph17228297

Dickerson, S. S., and Kemeny, M. E. (2004). Acute stressors and cortisol responses: a theoretical integration and synthesis of laboratory research. Psychol. Bull. 130, 355-391. doi: 10.1037/0033-2909.130.3.355

Djellel, D., Elena, F., and Panos, I. (2018). "Demographics and dynamics of mechanical turk workers," in Proceedings of the 11th ACM International Conference on Web Search and Data Mining, (Marina del Rey, CA: The RitzCarlton).

Durodié, B. (2020). Handling uncertainty and ambiguity in the COVID19 pandemic. Psychol. Trauma 12, S61-S62. doi: 10.1037/tra000 0713
Agency (JST)/Research Institute of Science and Technology for Society (RISTEX), Research grant from Japan-United States Brain Research Cooperative Program (AT), and Collaborative Research Network for Asian Children with Development Disorders under MEXT Policy Initiative (DH).

\section{ACKNOWLEDGMENTS}

We thank the staff at the Research Center for Child Mental Development and Department of Child and Adolescent Psychological Medicine, University of Fukui Hospital for their clerical support.

\section{SUPPLEMENTARY MATERIAL}

The Supplementary Material for this article can be found online at: https://www.frontiersin.org/articles/10.3389/fpsyg. 2021.782298/full\#supplementary-material

Elhadi, M., Alsoufi, A., Msherghi, A., Alshareea, E., Ashini, A., Nagib, T., et al. (2021). Psychological health, sleep quality, behavior, and internet use among people during the COVID-19 pandemic: a crosssectional study. Front. Psychiatry 12:632496. doi: 10.3389/fpsyt.2021.63 2496

Fegert, J. M., Vitiello, B., Plener, P. L., and Clemens, V. (2020). Challenges and burden of the Coronavirus 2019 (COVID-19) pandemic for child and adolescent mental health: a narrative review to highlight clinical and research needs in the acute phase and the long return to normality. Child Adolesc. Psychiatry Ment. Health 14:20. doi: 10.1186/s13034-020-00 329-3

Felitti, V. J., Anda, R. F., Nordenberg, D., Williamson, D. F., Spitz, A. M., Edwards, V., et al. (1998). Relationship of childhood abuse and household dysfunction to many of the leading causes of death in adults. The adverse childhood experiences (ACE) Study. Am. J. Prev. Med. 14, 245-258. doi: 10.1016/s07493797(98)00017-8

Francisco, R., Pedro, M., Delvecchio, E., Espada, J. P., Morales, A., Mazzeschi, C., et al. (2020). Psychological symptoms and behavioral changes in children and adolescents during the early phase of COVID-19 quarantine in three european countries. Front. Psychiatry 11:570164. doi: 10.3389/fpsyt.2020.57 0164

Gao, C., and Scullin, M. K. (2020). Sleep health early in the coronavirus disease 2019 (COVID-19) outbreak in the United States: integrating longitudinal, cross-sectional, and retrospective recall data. Sleep Med. 73, 1-10. doi: 10.1016/ j.sleep.2020.06.032

Gopal, A., Sharma, A. J., and Subramanyam, M. A. (2020). Dynamics of psychological responses to COVID-19 in India: a longitudinal study. PLoS One 15:e240650. doi: 10.1371/journal.pone.024 0650

Grumi, S., Provenzi, L., Accorsi, P., Biasucci, G., Cavallini, A., Decembrino, L., et al. (2021). Depression and anxiety in mothers who were pregnant during the COVID-19 outbreak in Northern Italy: the role of pandemic-related emotional stress and perceived social support. Front. Psychiatry 12:716488. doi: 10.3389/ fpsyt.2021.716488

Guo, J., Fu, M., Liu, D., Zhang, B., Wang, X., and Van Ijzendoorn, M. H. (2020). Is the psychological impact of exposure to COVID-19 stronger in adolescents with pre-pandemic maltreatment experiences? A survey of rural Chinese adolescents. Child Abuse Negl. 110(Pt 2), 104667. doi: 10.1016/j.chiabu.2020.10 4667

Hancock, J., and Mattick, K. (2020). Tolerance of ambiguity and psychological well-being in medical training: a systematic review. Med. Educ. 54, 125-137. doi: 10.1111/medu.14031 
Haque, M., Kumar, S., Charan, J., Bhatt, R., Islam, S., Dutta, S., et al. (2020). Utilisation, availability and price changes of medicines and protection equipment for COVID-19 among selected regions in India: findings and implications. Front. Pharmacol. 11:582154. doi: 10.3389/fphar.2020.58 2154

Heim, C., Newport, D. J., Heit, S., Graham, Y. P., Wilcox, M., Bonsall, R., et al. (2000). Pituitary-adrenal and autonomic responses to stress in women after sexual and physical abuse in childhood. JAMA 284, 592-597. doi: 10.1001/jama. 284.5.592

Higuchi, K. (2016). A two step approach to quantitative content analysis: KH coder tutorial using anne of green gables (PartI). Ritsumeikan Soc. Sci. Rev. 52, 77-91.

Higuchi, K. (2017). "A two step approach to quantitative content analysis: KH coder tutorial using anne of green gables (PartII)". Ritsumeikan Soc. Sci. Rev. $53,137-147$.

Hiraoka, D., Nishitani, S., Shimada, K., Kasaba, R., Fujisawa, T. X., and Tomoda, A. (2021). Epigenetic modification of the oxytocin gene is associated with gray matter volume and trait empathy in mothers. Psychoneuroendocrinology $\quad$ 123:105026. doi: 10.1016/j.psyneuen.2020.10 5026

Hiraoka, D., and Nomura, M. (2019). Maternal childhood adversity, OXTR genotype and cognitive load impact on perceptual and behavioral responses to infant crying. Psychoneuroendocrinology 104, 195-202. doi: 10.1016/j.psyneuen. 2019.03.005

Hiraoka, D., and Tomoda, A. (2020). Relationship between parenting stress and school closures due to the COVID-19 pandemic. Psychiatry Clin. Neurosci. 74, 497-498. doi: 10.1111/pcn.13088

International Monetary Fund (2021). World Economic Outlook [Online]. Available online at: https://www.imf.org/eternal/datamapper/ppppc@weo/oemdc/advec/ weoworld [Accessed 30 April 2021]

Jiao, W. Y., Wang, L. N., Liu, J., Fang, S. F., Jiao, F. Y., Pettoello-Mantovani, M., et al. (2020). Behavioral and emotional disorders in children during the COVID-19 epidemic. J. Pediatr. 221, 264.e261-266.e261. doi: 10.1016/j.jpeds. 2020.03.013

Kalia, V., Knauft, K., and Hayatbini, N. (2020). Cognitive flexibility and perceived threat from COVID-19 mediate the relationship between childhood maltreatment and state anxiety. PLoS One 15:e0243881. doi: 10.1371/journal. pone. 0243881

Kasaba, R., Shimada, K., and Tomoda, A. (2021). Neural mechanisms of parental communicative adjustments in spoken language. Neuroscience 457, 206-217. doi: 10.1016/j.neuroscience.2020. 12.002

Kuboshita, R., Fujisawa, T. X., Makita, K., Kasaba, R., Okazawa, H., and Tomoda, A. (2020). Intrinsic brain activity associated with eye gaze during mother-child interaction. Sci. Rep. 10:18903. doi: 10.1038/s41598-020-76 044-y

Lange, B. C. L., Callinan, L. S., and Smith, M. V. (2019). Adverse childhood experiences and their relation to parenting stress and parenting practices. Community Ment. Health J. 55, 651-662. doi: 10.1007/s10597-0180331-z

Lawson, M., Piel, M. H., and Simon, M. (2020). Child maltreatment during the COVID-19 pandemic: consequences of parental job loss on psychological and physical abuse towards children. Child Abuse Negl. 110(Pt 2), 104709. doi: 10.1016/j.chiabu.2020.104709

Lebel, C., Mackinnon, A., Bagshawe, M., Tomfohr-Madsen, L., and Giesbrecht, G. (2020). Elevated depression and anxiety symptoms among pregnant individuals during the COVID-19 pandemic. J. Affect Disord. 277, 5-13. doi: 10.1016/j.jad. 2020.07.126

Lee, S. A. (2020). Coronavirus anxiety scale: a brief mental health screener for COVID-19 related anxiety. Death Stud. 44, 393-401. doi: 10.1080/07481187. 2020.1748481

Lee, S. J., Ward, K. P., Lee, J. Y., and Rodriguez, C. M. (2021). Parental social isolation and child maltreatment risk during the COVID-19 pandemic. J. Fam. Violence Online ahead of print doi: 10.1007/s10896-020-00 244-3

Lena, H., Mareike, B., Stefan, M., and Armin, S. (2020). Problems and pitfalls of retrospective survey questions in COVID-19 studies. Surv. Res. Methods 14, 109-114.
Levey, E. J., Apter, G., and Harrison, A. M. (2017). The global problem of child maltreatment: perspectives on mechanisms of influence and illness presentation. Int. J. Cult. Ment. Health 10, 90-96. doi: 10.1080/17542863.2016. 1264440

Malhi, P., Bharti, B., and Sidhu, M. (2021). Stress and parenting during the COVID-19 pandemic: psychosocial impact on children. Indian J. Pediatr. 88:481. doi: 10.1007/s12098-021-03 665-0

Malkawi, S. H., Almhdawi, K., Jaber, A. F., and Alqatarneh, N. S. (2021). COVID19 quarantine-related mental health symptoms and their correlates among mothers: a cross sectional Study. Matern. Child Health J. 25, 695-705. doi: 10.1007/s10995-020-03034-x

Marchetti, D., Fontanesi, L., Mazza, C., Di Giandomenico, S., Roma, P., and Verrocchio, M. C. (2020). Parenting-related exhaustion during the Italian COVID-19 lockdown. J. Pediatr. Psychol. 45, 1114-1123. doi: 10.1093/jpepsy/ jsaa093

Merrick, M. T., Ford, D. C., Ports, K. A., and Guinn, A. S. (2018). Prevalence of Adverse Childhood experiences from the 2011-2014 behavioral risk factor surveillance system in 23 states. JAMA Pediatr. 172, 1038-1044. doi: 10.1001/ jamapediatrics.2018.2537

Nazif-Muñoz, J. I., Peña, S., and Oulhote, Y. (2021). The global viralization of policies to contain the spreading of the COVID-19 pandemic: analyses of school closures and first reported cases. PLoS One 16:e248828. doi: 10.1371/journal. pone. 0248828

Park, C., Rosenblat, J. D., Brietzke, E., Pan, Z., Lee, Y., Cao, B., et al. (2019). Stress, epigenetics and depression: a systematic review. Neurosci. Biobehav. Rev. 102, 139-152. doi: 10.1016/j.neubiorev.2019.04.010

R Core Team (2019). R: A Language and Environment for Statistical Computing. Vienna: R Foundation for Statistical Computing.

Ramaswamy, S., and Seshadri, S. (2020). Children on the brink: risks for child protection, sexual abuse, and related mental health problems in the COVID19 pandemic. Indian J. Psychiatry 62, S404-S413. doi: 10.4103/psychiatry. IndianJPsychiatry_1032_20

Robillard, R., Dion, K., Pennestri, M. H., Solomonova, E., Lee, E., Saad, M., et al. (2021). Profiles of sleep changes during the COVID-19 pandemic: demographic, behavioural and psychological factors. J. Sleep Res. 30:e13231. doi: $10.1111 /$ jsr.13231

Rosseel, Y. (2012). lavaan: An $\mathrm{R}$ package for structural equation modeling. J. Stat. Softw. 48, 1-36. doi: 10.3389/fpsyg.2014. 01521

Sharma, B. R., Gupta, M., Sharma, A. K., Sharma, S., Gupta, N., Relhan, N., et al. (2007). Suicides in Northern India: comparison of trends and review of literature. J. Forensic Leg. Med. 14, 318-326. doi: 10.1016/j.jcfm.2006. 08.009

Shimada, K., Kasaba, R., Fujisawa, T. X., Sakakibara, N., Takiguchi, S., and Tomoda, A. (2018). Subclinical maternal depressive symptoms modulate right inferior frontal response to inferring affective mental states of adults but not of infants. J. Affect Disord. 229, 32-40. doi: 10.1016/j.jad.2017. 12.031

Shimada, K., Kasaba, R., Yao, A., and Tomoda, A. (2019). Less efficient detection of positive facial expressions in parents at risk of engaging in child physical abuse. BMC Psychol. 7:56. doi: 10.1186/s40359-0190333-9

Spinelli, M., Lionetti, F., Pastore, M., and Fasolo, M. (2020). Parents' stress and children's psychological problems in families facing the COVID19 outbreak in Italy. Front. Psychol. 11:1713. doi: 10.3389/fpsyg.2020. 01713

Stoet, G. (2010). PsyToolkit: a software package for programming psychological experiments using Linux. Behav. Res. Methods 42, 1096-1104. doi: 10.3758/ BRM.42.4.1096

Stoet, G. (2017). PsyToolkit: a novel web-based method for running online questionnaires and reaction-time experiments. Teach. Psychol. 44, 24-31.

Tang, S., Xiang, M., Cheung, T., and Xiang, Y. T. (2021). Mental health and its correlates among children and adolescents during COVID-19 school closure: the importance of parent-child discussion. J. Affect Disord. 279, 353-360. doi: 10.1016/j.jad.2020.10.016 
Tso, W. W. Y., Wong, R. S., Tung, K. T. S., Rao, N., Fu, K. W., Yam, J. C. S., et al. (2020). Vulnerability and resilience in children during the COVID19 pandemic. Eur. Child Adolesc. Psychiatry Online ahead of print doi: 10.1007/s00787-020-01680-8

Uddin, J., Alharbi, N., Uddin, H., Hossain, M. B., Hatipoğlu, S. S., Long, D. L., et al. (2020). Parenting stress and family resilience affect the association of adverse childhood experiences with children's mental health and attention-deficit/hyperactivity disorder. J. Affect Disord. 272, 104-109. doi: 10.1016/j.jad.2020.03. 132

UNICEF (2021). COVID-19 and School Closures: One year of education disruption [Online]. Available online at: https://data.unicef.org/resources/one-year-ofcovid-19-and-school-closures/ [Accessed 30 April 2021].

World Health Organization (2021). Coronavirus diseases (COVID-19) Weekly Epidemiological Update and Weekly Operational Update [Online]. Available online at: https://www.who.int/emergencies/diseases/novel-coronavirus-2019/ situation-reports [Accessed 30 April 2021]
Conflict of Interest: The authors declare that the research was conducted in the absence of any commercial or financial relationships that could be construed as a potential conflict of interest.

Publisher's Note: All claims expressed in this article are solely those of the authors and do not necessarily represent those of their affiliated organizations, or those of the publisher, the editors and the reviewers. Any product that may be evaluated in this article, or claim that may be made by its manufacturer, is not guaranteed or endorsed by the publisher.

Copyright $\odot 2021$ Kurata, Hiraoka, Ahmad Adlan, Jayanath, Hamzah, AhmadFauzi, Fujisawa, Nishitani and Tomoda. This is an open-access article distributed under the terms of the Creative Commons Attribution License (CC BY). The use, distribution or reproduction in other forums is permitted, provided the original author(s) and the copyright owner(s) are credited and that the original publication in this journal is cited, in accordance with accepted academic practice. No use, distribution or reproduction is permitted which does not comply with these terms. 